\title{
Query Answering System of Shahih Hadith Muttafaqun 'Alaih Using Indonesian Thesaurus Based on Query Expansion and Naïve Bayes Classifier
}

\author{
Muhammad Fairuz Zumar Rounaqi, Cahyo Crysdian, Roro Inda Melani
}

\begin{abstract}
Hadith are all the words, deeds and provisions of the Prophet Muhammad SAW that are used as the second of Islamic law after Al-Quran. The purpose of this study is to make an Information Retrieval system called the Query Answering System is expected to facilitate users in searching and finding the hadith documents as the user's needs. This study implements the Naïve Bayes Classifier method combined with Indonesian thesaurus as a query expansion to find the hadith documents that relevant to the input query. Based on the testing of 50 query data, the test results show that the use of query expansion gives better results than without using query expansion. Where based on testing of the top 1 data without using query expansion obtained an average recall value of $62 \%$, an average precision value of $62 \%$, an average accuracy value of $92.4 \%$ and an average value of the f-measure of $62 \%$, while testing using query expansion obtained an average recall value of $66 \%$, an average precision value of $66 \%$, an average accuracy value of 93.2\% and an average $f$-measure value of $66 \%$. Based on the test results, the use of query expansion shows an improvement in the average recall value of $4 \%$, an improvement in the average precision value of $4 \%$, and an improvement in the average accuracy value of $0.8 \%$ and an improvement in the average f-measure value of $4 \%$ compared on without using query expansion.
\end{abstract}

Index Terms - hadith, information retrieval, query expansion, naïve bayes.

\section{INTRODUCTION}

$\mathrm{H}$ ADITH is the second source of Islamic law after AlQur'an. Al-Hafidz Ibnu Hajar Al Asqalany explained in the book of Bulughul Maram that the hadith are all the words, deeds and decrees of the prophet Muhammad SAW which are used as provisions or Islamic law [1]. In general, the Hadith is in line with Al- Qur'an, where the hadith explains the mubham, details the mujmal limits the absolute, specifies the

Manuscript received - Desember, 2019. This work was supported in part by Computer Science Department of Maulana Malik Ibrahim Islamic State University.

Muhammad Fairuz Zumar Rounaqi, Cahyo Crysdian, Roro Inda Melani are the researcher of Computer Science Departement of Maulana Malik Ibrahim Islamic State University, Malang, Indonesia general and elaborates on the laws and the aims of its purpose.

The hadith that can be used as a way of life is hadith that claims it is truth by the Ulama' of hadith experts, the one type of it is the shahih hadith narrated by Imam Bukhari and Imam Muslim. Shahih hadith is widely used by Ulama to determine the law of certain disputes. One of the books of the collection of authentic hadith written by Imam Bukhari and Imam Muslim is the book of Al-Lu'Lu' Wal Marjan by Muhammad Fuad Bin Abdul Baqi. To facilitate the users for finding hadith documents from that book and accommodate with the user needs, it is important to develop an Information Retrieval system as a simple data search system where users only posed a query to the system, then the system will look for hadith that match from the database and displays search results to the users.

To find a relevant hadith, it is necessary to measure the similarity between queries and hadith documents. This study implement the naïve bayes method to measure the probability of queries on hadith documents. Then the results of probability testing will be ranked and sorted from the highest probability value, and then the system will displays the hadith data based on the rank results as a feedback from the query posed by the users.

\section{RELATED RESEARCH}

Ginting \& Trinada [2] examines the implementation of the Naïve Bayes Classifier method as a technique for constructing a classification model based on documents contained in libraries. In their research, the Naïve Bayes Classifier method is used to classify several titles and categories of documents that are already in the library database, then the search process is carried out by involving descriptions of each document so that it can display more references as search results. The results of this study, the information retrieval system that has been made can display more documents based on user queries so that users get more document references. However, this research does not measure the amount of recall and precision of the documents retrieved, so it is not known how much the percentage of relevant documents to the 
user query.

Yadav[3] applies the documents similarity matrix and Naïve Bayes classification to do web information retrieval. In this research, the document-document similarity matrix was used after performing preprocessing and feature construction. Then, a Naïve Bayes Classifier was used to find the relevant category of information that is required for the user. The purpose of this research is to analyze the proposed algorithm with sensitivity, specificity, and accuracy. The result of this research is the proposed algorithm can increase 3\% of sensitivity as compared with the existing algorithm. Also, it increased the specificity and accuracy as compared with the existing algorithm.

Shahabadkar, et al. [4] proposes a new Enhanced Query Expansion based Classifier (EQC) technique for web document retrieval. Where expansion of demand is used to improve document retrieval. Original user requests are reformulated and feedback is given to the dataset to find relevant documents. The results of this study showed an increase in the value of precision by $1 \%$, an increase in the value of recall by $3 \%$ and an increase in the value of the f-measure by $3.5 \%$. That is measurements of precision, recall, and f-measure show an increase in document retrieval schemes relevant to reduced computational complexity.

\section{NAÏVE BAYES CLASSIFIER}

This study proposed the Naïve Bayes Classifier Method. That method will implemented on the searching process to found the relevant hadith. According to Ginting \& Trinada [2], Naïve Bayes Classifier is one of machine learning method that utilizes the probability and statistical calculations proposed by Thomas Bayes. According to Suyanto [5], probability or conditional probability is expressed as comparison 1:

$$
\mathrm{P}(\mathrm{H} \mid \mathrm{X})=\mathrm{P}(\mathrm{X} \mid \mathrm{H}) * \mathrm{P}(\mathrm{H}) / \mathrm{p}(\mathrm{X})
$$

Where $\mathrm{X}$ is proof, $\mathrm{H}$ is the hypothesis, $\mathrm{P}(\mathrm{H} \mid \mathrm{X})$ is the probability that the hypothesis $\mathrm{H}$ is true for the proof of $\mathrm{X}$ in other words $\mathrm{P}(\mathrm{H} \mid \mathrm{X})$ is the posterior probability $\mathrm{H}$ with terms $\mathrm{X}, \mathrm{P}(\mathrm{H} \mid \mathrm{X})$ is the probability that the proof of $\mathrm{X}$ is true for the hypothesis $\mathrm{H}$ or probability $\mathrm{X}$ with the condition $\mathrm{H}, \mathrm{P}(\mathrm{H})$ is the probability of the prior hypothesis $\mathrm{H}$, and $\mathrm{P}(\mathrm{X})$ is the probability of prior proof of X.

\section{RESEARCH METHODOLOGY}

\section{A. Data Collection}

The data used in this study was taken from the book Al Lu 'Lu' Wal Marjan which contains a collection of Sahih hadith narrated by Imam Bukhari and Imam Muslim by Muhammad Fuad Bin Abdul Baqi. The hadith topics taken from the book has the follow Indonesian term such as "Iman, Thaharah, Haidh,
Shalat, Mushala, Shalat Orang Musafir, Al-Jum'ah, Shalat Dua Hari Raya, Shalat Istisqaa ', Salat Gerhana, Janazah".

\section{B. Process Design}

The process design purposes to create a flow system that would be implemented to this study, an overview of the process design shown in Figure 1.

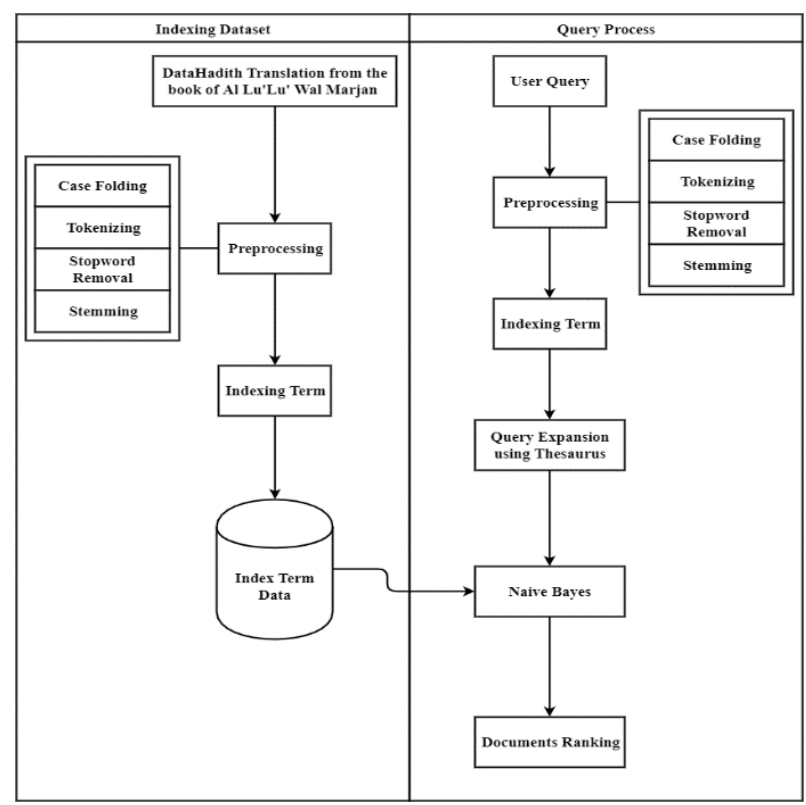

Figure 1. Design Process

Based on Figure 4.1, the searching process started from preprocessing query posed by the users, the preprocessing steps are contains case folding process, tokenizing process, stopword removal process, and stemming. After that, the terms of query as the results of preprocessing will be expanded based on Indonesian thesaurus, then the system will match the terms of query with the terms of documents. The result of matching terms will be build into the training set, it used to facilitate the system for calculate the probability value. Then the results of probability calculation will be ranked and showed to the users as the result of searching process.

\section{Experiment}

The experiment done by evaluating the results of testing. According to Hasugian [6], there are two things that can be used as a reference assessment to measure the effectiveness of an information retrieval, that is precision and recall. The confusion matrix of recall, precision, and accuracy is given by Table 1 .

Table 1. Confusion matrix of recall, precision, and accuracy

\begin{tabular}{|l|l|l|l|}
\cline { 2 - 4 } \multicolumn{1}{c|}{} & Relevant & Irrelevant & Total \\
\hline Retrieve & $\mathrm{a}$ (hits) & $\mathrm{b}$ (noise) & $\mathrm{a}+\mathrm{b}$ \\
\hline Not Retrieve & $\mathrm{c}$ (misses) & $\mathrm{d}$ (rejected) & $\mathrm{c}+\mathrm{d}$ \\
\hline Total & $\mathrm{a}+\mathrm{c}$ & $\mathrm{b}+\mathrm{d}$ & $\mathrm{a}+\mathrm{b}+\mathrm{c}+\mathrm{d}$ \\
\hline
\end{tabular}

Description of Table above:

Recall $=[a /(a+c)] \times 100 \%$

Precision $=[a /(a+b)] \times 100 \%$

Accuracy $=[a+d /(a+b+c+d)] \times 100 \%$ 
To evaluate the information retrieval system also necessary to measure the f-measure. Where the fmeasure calculation is used to evaluate the information retrieval system by combining the results of precision and recall calculations. The F-measure represents the relative effect between precision and recall. F-measure is the mean harmonic weight of precision and recall. Below is the formula of the f-measure calculation. Precision $\mathrm{x}$ Recall

F-Measure $=2 \mathrm{x}$

Precision + Recall

\section{RESUlTS AND DISCUSSION}

The first test is system testing that has been done by posting the 50 queries into the system, and the system provides feedback in the form of relevant hadith documents. The 50 query testing is given by Table 2 .

Table 2. List of query test

\begin{tabular}{|c|c|c|}
\hline \multicolumn{3}{|c|}{ Table of Query Test } \\
\hline No & Query in Indonesian term & $\begin{array}{c}\text { Query expansion in } \\
\text { Indonesian term }\end{array}$ \\
\hline 1 & Rukun Iman & $\begin{array}{l}\text { Rukun Iman Tiang } \\
\text { Religiositas }\end{array}$ \\
\hline 2 & Rukun Islam & Rukun Islam Tiang \\
\hline 3 & Menghormati Tetangga & $\begin{array}{l}\text { Hormat Tetangga Segan } \\
\text { Jiran }\end{array}$ \\
\hline 4 & Tanda Orang Munafik & $\begin{array}{l}\text { Tanda Munafik Ciri } \\
\text { Munafiq }\end{array}$ \\
\hline 5 & Meratakan Saf Saat Shalat & Saf Shalat Jajar Salat \\
\hline 6 & Tanda Orang Beriman & $\begin{array}{l}\text { Tanda Iman Ciri } \\
\text { Religiositas }\end{array}$ \\
\hline 7 & Hukum Bunuh Diri & Hukum Bunuh Atur \\
\hline 8 & Syafaat Nabi & Syafaat Nabi Mediasi \\
\hline 9 & Ahli Surga & Ahli Surga Kaum Janah \\
\hline 10 & Ahli Neraka & Ahli Neraka Kaum \\
\hline 11 & $\begin{array}{l}\text { Anjuran Bersuci Sebelum } \\
\text { Shalat }\end{array}$ & Suci Shalat Murni Salat \\
\hline 12 & Anjuran Bersiwak & Siwak \\
\hline 13 & Hukum Jilatan Anjing & Hukum Jilat Anjing Atur \\
\hline 14 & Hukum Kencing Bayi & $\begin{array}{l}\text { Hukum Kencing Bayi } \\
\text { Atur Ompol }\end{array}$ \\
\hline 15 & $\begin{array}{l}\text { Hukum Menggauli Istri Saat } \\
\text { Sedang Haidh }\end{array}$ & $\begin{array}{l}\text { Hukum Gaul Istri Haidh } \\
\text { Atur }\end{array}$ \\
\hline 16 & Tata Cara Mandi Janabat & $\begin{array}{l}\text { Tata Mandi Janabat } \\
\text { Siram Junub }\end{array}$ \\
\hline 17 & Menjaga Aurat & Jaga Aurat Tutup \\
\hline 18 & Cara Tayammum & Tayammum \\
\hline 19 & Fadilat Shalat Jama'ah & $\begin{array}{l}\text { Fadilat Shalat Jamaah } \\
\text { Rahmat Salat }\end{array}$ \\
\hline 20 & $\begin{array}{l}\text { Larangan Kencing dalam Air } \\
\text { yang Menggenang }\end{array}$ & $\begin{array}{l}\text { Larang Kencing Air } \\
\text { Genang Ompol }\end{array}$ \\
\hline 21 & $\begin{array}{l}\text { Larangan Berjalan didepan } \\
\text { Orang Shalat }\end{array}$ & $\begin{array}{l}\text { Larang Jalan Shalat } \\
\text { Salat }\end{array}$ \\
\hline 22 & Dosa Besar & Dosa Maksiat \\
\hline 23 & Bacaan dalam Shalat & Baca Shalat Salat \\
\hline 24 & $\begin{array}{l}\text { Kewajiban Beriman kepada } \\
\text { Allah }\end{array}$ & $\begin{array}{l}\text { Wajib Iman Allah Kudu } \\
\text { Religiositas }\end{array}$ \\
\hline 25 & $\begin{array}{l}\text { Kewajiban Beriman kepada } \\
\text { Rasulullah }\end{array}$ & $\begin{array}{l}\text { Wajib Iman Rasulullah } \\
\text { Kudu Religiositas }\end{array}$ \\
\hline 26 & Adab Buang Air & Adab Buang Air Akhlak \\
\hline 27 & $\begin{array}{l}\text { Cara Membersihkan Kulit } \\
\text { Bangkai }\end{array}$ & $\begin{array}{l}\text { Bersih Kulit Bangkai } \\
\text { Suci }\end{array}$ \\
\hline 28 & Amal yang Utama & Amal Utama Kebaji Baik \\
\hline 29 & $\begin{array}{l}\text { Mengangkat Kedua Tangan } \\
\text { pada saat Takbiratul Ihram }\end{array}$ & $\begin{array}{l}\text { Angkat Tangan } \\
\text { Takbiratul Ihram Naik }\end{array}$ \\
\hline 30 & Cara Berwudhu & Wudhu Suci \\
\hline 31 & Larangan Bicara Ketika Shalat & Larang Bicara Shalat \\
\hline
\end{tabular}

\begin{tabular}{|l|l|l|}
\hline & & Salat \\
\hline 32 & $\begin{array}{l}\text { Bacaan Dalam Ruku' dan } \\
\text { Sujud }\end{array}$ & Baca Ruku Sujud Sembah \\
\hline 33 & Shalatnya Orang Musafir & Shalat Musafir Salat \\
\hline 34 & Fadilat Menghafal Qur'an & $\begin{array}{l}\text { Fadilat Hafal } \text { Quran } \\
\text { Rahmat }\end{array}$ \\
\hline 35 & Shalat Sunnat & Shalat Sunnat Salat \\
\hline 36 & Shalat Dua Hari Raya & Shalat Raya Salat \\
\hline 37 & Shalat Malam & Shalat Malam Salat \\
\hline 38 & Menjamak Shalat & Jamak Shalat Salat \\
\hline 39 & Cara Sujud Tilawah & Sujut Tilawah Sembah \\
\hline 40 & Perubahan Arah Qiblat & Rubah Arah Ganti \\
\hline 41 & Shalat Sunnat yang dilarang & $\begin{array}{l}\text { Shalat Sunnat Larang } \\
\text { Salat }\end{array}$ \\
\hline 42 & Hukum Shalat Memakai Sepatu & $\begin{array}{l}\text { Hukum Shalat Sepatu } \\
\text { Atur Salat }\end{array}$ \\
\hline 43 & Imam Shalat & $\begin{array}{l}\text { Imam Shalat Pimpin } \\
\text { Salat }\end{array}$ \\
\hline 44 & Meminta Hujan & Hujan \\
\hline 45 & Membersihkan Najis & Bersih Najis Suci \\
\hline 46 & Wajib Mandi & Wajib Mandi Kudu Siram \\
\hline 47 & Shalat Gerhana & Shalat Gerhana Salat \\
\hline 48 & Shalat di Kendaraan & Shalat Kendara Salat \\
\hline 49 & Memandikan Orang Mati & Mandi Mati Siram \\
\hline 50 & Ingat Mati & Mati Maut \\
\hline &
\end{tabular}

The result of system without query expansion is given by Table 3 .

Table 3. Result of system testing without query expansion

\begin{tabular}{|c|c|c|c|c|c|c|c|c|c|c|}
\hline \multicolumn{11}{|c|}{ RESULT OF SYSTEM TESTING WITHOUT QUERY EXPANSION } \\
\hline \multirow{2}{*}{ Query } & \multicolumn{10}{|c|}{ Ranked result in term of hadith number } \\
\hline & 1 & 2 & 3 & 4 & 5 & 6 & 7 & 8 & 9 & 10 \\
\hline $\mathrm{Q}-1$ & 36 & 97 & 29 & 28 & 22 & 21 & 47 & 23 & 30 & 34 \\
\hline $\mathrm{Q}-2$ & 9 & 6 & 25 & 24 & 77 & 75 & 63 & 64 & 62 & 89 \\
\hline $\mathrm{Q}-3$ & 29 & 30 & 53 & 88 & 471 & 370 & 400 & 509 & 23 & 403 \\
\hline $\mathrm{Q}-4$ & 38 & 47 & 28 & 48 & 37 & 49 & 194 & 370 & 471 & 23 \\
\hline $\mathrm{Q}-5$ & 248 & 282 & 251 & 506 & 403 & 400 & 342 & 294 & 508 & 510 \\
\hline Q-6 & 47 & 28 & 48 & 36 & 97 & 29 & 49 & 22 & 21 & 23 \\
\hline $\mathrm{Q}-7$ & 95 & 61 & 70 & 69 & 175 & 55 & 85 & 73 & 96 & 163 \\
\hline Q-8 & 122 & 121 & 126 & 125 & 120 & 66 & 215 & 118 & 119 & 115 \\
\hline Q-9 & 8 & 114 & 72 & 71 & 102 & 115 & 113 & 127 & 369 & 33 \\
\hline Q-10 & 72 & 116 & 127 & 114 & 71 & 117 & 133 & 115 & 16 & 102 \\
\hline Q-11 & 269 & 420 & 419 & 421 & 134 & 268 & 192 & 386 & 270 & 422 \\
\hline $\mathrm{Q}-12$ & 143 & 144 & 490 & 142 & 269 & 422 & 419 & 491 & 492 & 421 \\
\hline Q-13 & 160 & 175 & 96 & 370 & 471 & 163 & 290 & 291 & 403 & 23 \\
\hline Q-14 & 163 & 164 & 190 & 155 & 161 & 442 & 175 & 162 & 96 & 370 \\
\hline Q-15 & 169 & 168 & 171 & 173 & 174 & 170 & 172 & 253 & 179 & 197 \\
\hline Q-16 & 183 & 171 & 176 & 177 & 178 & 181 & 197 & 179 & 198 & 196 \\
\hline $\mathrm{Q}-17$ & 195 & 422 & 369 & 312 & 367 & 194 & 368 & 104 & 533 & 370 \\
\hline Q-18 & 207 & 206 & 208 & 396 & 209 & 14 & 11 & 10 & 566 & 7 \\
\hline Q-19 & 381 & 387 & 383 & 350 & 380 & 353 & 377 & 351 & 447 & 388 \\
\hline Q-20 & 161 & 151 & 163 & 543 & 154 & 186 & 164 & 153 & 150 & 204 \\
\hline $\mathrm{Q}-21$ & 285 & 284 & 317 & 283 & 286 & 282 & 400 & 370 & 403 & 475 \\
\hline $\mathrm{Q}-22$ & 118 & 76 & 81 & 536 & 62 & 120 & 284 & 389 & 53 & 80 \\
\hline $\mathrm{Q}-23$ & 265 & 260 & 266 & 264 & 263 & 450 & 341 & 224 & 262 & 256 \\
\hline $\mathrm{Q}-24$ & 12 & 94 & 11 & 19 & 18 & 93 & 29 & 8 & 10 & 134 \\
\hline $\mathrm{Q}-25$ & 12 & 94 & 11 & 18 & 27 & 19 & 10 & 50 & 29 & 83 \\
\hline $\mathrm{Q}-26$ & 149 & 148 & 150 & 154 & 157 & 158 & 172 & 153 & 186 & 185 \\
\hline $\mathrm{Q}-27$ & 205 & 189 & 471 & 370 & 400 & 23 & 509 & 403 & 186 & 294 \\
\hline Q-28 & 50 & 51 & 25 & 24 & 75 & 429 & 77 & 52 & 369 & 381 \\
\hline $\mathrm{Q}-29$ & 217 & 218 & 516 & 317 & 244 & 349 & 277 & 370 & 471 & 143 \\
\hline Q-30 & 135 & 136 & 437 & 140 & 176 & 204 & 159 & 178 & 181 & 141 \\
\hline Q-31 & 323 & 312 & 311 & 317 & 475 & 473 & 351 & 476 & 474 & 350 \\
\hline Q-32 & 220 & 275 & 272 & 521 & 234 & 224 & 232 & 217 & 341 & 522 \\
\hline Q-33 & 400 & 398 & 402 & 399 & 401 & 342 & 362 & 508 & 403 & 354 \\
\hline Q-34 & 453 & 452 & 460 & 454 & 472 & 304 & 461 & 451 & 93 & 259 \\
\hline Q-35 & 480 & 447 & 413 & 474 & 420 & 399 & 362 & 414 & 421 & 477 \\
\hline Q-36 & 508 & 509 & 507 & 506 & 510 & 511 & 278 & 505 & 342 & 362 \\
\hline $\mathrm{Q}-37$ & 436 & 432 & 428 & 433 & 439 & 374 & 431 & 404 & 406 & 438 \\
\hline Q-38 & 411 & 409 & 410 & 342 & 508 & 362 & 400 & 403 & 398 & 354 \\
\hline Q-39 & 341 & 338 & 340 & 339 & 277 & 246 & 276 & 335 & 272 & 220 \\
\hline Q-40 & 303 & 302 & 407 & 406 & 250 & 31 & 304 & 291 & 320 & 150 \\
\hline Q-41 & 473 & 474 & 475 & 351 & 476 & 477 & 350 & 310 & 420 & 421 \\
\hline Q-42 & 325 & 155 & 403 & 400 & 370 & 342 & 294 & 374 & 508 & 509 \\
\hline Q-43 & 235 & 482 & 239 & 503 & 270 & 354 & 243 & 268 & 238 & 233 \\
\hline Q-44 & 517 & 46 & 518 & 405 & 515 & 404 & 5 & 384 & 11 & 566 \\
\hline Q-45 & 189 & 210 & 166 & 349 & 162 & 167 & 100 & 471 & 370 & 23 \\
\hline Q-46 & 487 & 199 & 196 & 489 & 180 & 488 & 175 & 197 & 490 & 198 \\
\hline $\mathrm{Q}-47$ & 530 & 527 & 526 & 529 & 522 & 528 & 524 & 523 & 525 & 342 \\
\hline Q-48 & 406 & 407 & 279 & 408 & 313 & 282 & 232 & 342 & 301 & 362 \\
\hline Q-49 & 546 & 544 & 545 & 487 & 489 & 58 & 85 & 534 & 555 & 185 \\
\hline Q-50 & 72 & 85 & 560 & 537 & 530 & 58 & 71 & 60 & 66 & 65 \\
\hline
\end{tabular}


And the result of system testing with query expansion is given by Table 4 .

Table 4. Result of system testing with query expansion

\begin{tabular}{|c|c|c|c|c|c|c|c|c|c|c|}
\hline \multirow{3}{*}{ Query } & \multicolumn{10}{|c|}{ RESULT OF SYSTEM TESTING WITH QUERY EXPANSION } \\
\hline & \multicolumn{10}{|c|}{ Rank Result in Term of Hadith Number } \\
\hline & 1 & 2 & 3 & 4 & 5 & 6 & 7 & 8 & 9 & $\mathbf{1 0}$ \\
\hline Q-1 & 23 & 28 & 22 & 47 & 83 & 471 & 370 & 29 & 32 & 36 \\
\hline $\mathrm{Q}-2$ & 9 & 24 & 25 & 63 & 64 & 6 & 75 & 77 & 89 & 479 \\
\hline Q-3 & 29 & 30 & 471 & 370 & 509 & 23 & 400 & 403 & 294 & 67 \\
\hline Q-4 & 38 & 47 & 48 & 37 & 28 & 370 & 471 & 400 & 23 & 403 \\
\hline Q-5 & 510 & 400 & 403 & 370 & 282 & 342 & 294 & 248 & 508 & 509 \\
\hline Q-6 & 47 & 28 & 23 & 48 & 22 & 83 & 471 & 370 & 29 & 32 \\
\hline Q-7 & 95 & 55 & 175 & 69 & 70 & 61 & 96 & 471 & 370 & 163 \\
\hline Q-8 & 122 & 121 & 126 & 125 & 294 & 370 & 471 & 215 & 66 & 400 \\
\hline Q-9 & 519 & 395 & 72 & 8 & 146 & 370 & 393 & 471 & 369 & 113 \\
\hline $\mathrm{Q}-10$ & 72 & 127 & 116 & 115 & 40 & 395 & 117 & 71 & 519 & 394 \\
\hline Q-11 & 134 & 403 & 400 & 370 & 342 & 294 & 192 & 386 & 508 & 509 \\
\hline Q-12 & 143 & 144 & 142 & 490 & 493 & 492 & 491 & 375 & 376 & 379 \\
\hline $\mathrm{Q}-13$ & 160 & 370 & 471 & 400 & 509 & 175 & 23 & 403 & 294 & 186 \\
\hline Q-14 & 163 & 164 & & & & & & & & \\
\hline Q-15 & 169 & 168 & 171 & 174 & 170 & 173 & & & & \\
\hline Q-16 & 177 & 183 & 171 & 181 & 210 & 179 & 176 & 197 & 182 & 546 \\
\hline Q-17 & 549 & 295 & 422 & 195 & 369 & 471 & 370 & 112 & 312 & 23 \\
\hline Q-18 & 207 & 206 & 208 & 396 & 209 & 14 & 11 & 10 & 566 & 7 \\
\hline Q-19 & 381 & 400 & 403 & 350 & 370 & 383 & 377 & 342 & 294 & 380 \\
\hline $\mathrm{Q}-20$ & 161 & 151 & 543 & 186 & 163 & 154 & 155 & & & \\
\hline $\mathrm{Q}-21$ & 284 & 282 & 317 & 285 & 475 & 388 & 403 & 400 & 370 & 473 \\
\hline $\mathrm{Q}-22$ & 80 & 53 & 364 & 81 & 324 & 389 & 435 & 284 & 231 & 229 \\
\hline $\mathrm{Q}-23$ & 260 & 265 & 264 & 225 & 263 & 522 & 450 & 341 & 222 & 504 \\
\hline $\mathrm{Q}-24$ & 12 & 29 & 83 & 11 & 134 & 94 & 23 & 344 & 19 & 93 \\
\hline $\mathrm{Q}-25$ & 12 & 27 & 83 & 197 & 23 & 490 & 509 & 19 & 28 & 549 \\
\hline Q-26 & 149 & 148 & 150 & 154 & 157 & 186 & 153 & 158 & 172 & 185 \\
\hline Q-27 & 205 & 189 & 154 & 152 & 370 & 471 & 134 & 400 & 403 & 23 \\
\hline Q-28 & 50 & 24 & 25 & 77 & 51 & 467 & 75 & 370 & 471 & 550 \\
\hline Q-29 & 217 & 218 & 516 & 317 & & & & & & \\
\hline Q-30 & 135 & 136 & 437 & 140 & 176 & 204 & 159 & 178 & 181 & 141 \\
\hline Q-31 & 317 & 323 & 312 & 475 & 311 & 400 & 403 & 370 & 473 & 342 \\
\hline $\mathrm{Q}-32$ & 272 & 275 & 220 & 234 & 521 & 341 & 273 & 232 & 340 & 217 \\
\hline Q-33 & 400 & 398 & 402 & 401 & 399 & 510 & 403 & 366 & 342 & 294 \\
\hline Q-34 & 451 & 453 & 471 & 370 & 509 & 403 & 23 & 400 & 452 & 294 \\
\hline Q-35 & 477 & 420 & 421 & 414 & 480 & 474 & 422 & 413 & 399 & 445 \\
\hline Q-36 & 510 & 509 & 508 & 507 & 506 & 403 & 400 & 366 & 342 & 294 \\
\hline Q-37 & 432 & 439 & 433 & 428 & 436 & 431 & 374 & 441 & 404 & 427 \\
\hline Q-38 & 411 & 409 & 410 & 342 & 362 & 508 & 398 & 400 & 403 & 354 \\
\hline Q-39 & 338 & 341 & 340 & 339 & 277 & 276 & 246 & 272 & 293 & 318 \\
\hline Q-40 & 303 & 302 & 407 & 237 & 250 & 406 & 488 & 471 & 370 & 31 \\
\hline Q-41 & 477 & 475 & 473 & 474 & 476 & 351 & 350 & 420 & 317 & 421 \\
\hline $\mathrm{Q}-42$ & 155 & 403 & 370 & 400 & 342 & 294 & 279 & 265 & 439 & 295 \\
\hline Q-43 & 503 & 269 & 268 & 502 & 566 & 400 & 403 & 354 & 270 & 370 \\
\hline Q-44 & 517 & 46 & 518 & 405 & 515 & 404 & 5 & 384 & 11 & 566 \\
\hline Q-45 & 189 & 162 & 154 & 152 & 134 & 370 & 471 & 163 & 166 & 386 \\
\hline Q-46 & 487 & 489 & 197 & 488 & 490 & 199 & 175 & 183 & 198 & 196 \\
\hline Q-47 & 527 & 530 & 529 & 526 & 528 & 522 & 523 & 510 & 525 & 400 \\
\hline Q-48 & 406 & 279 & 407 & 510 & 282 & 408 & 400 & 403 & 366 & 342 \\
\hline Q-49 & 546 & 183 & 544 & 487 & 186 & 485 & 184 & 181 & 549 & 171 \\
\hline Q-50 & 58 & 85 & 534 & 555 & 530 & 65 & 556 & 549 & 59 & 546 \\
\hline
\end{tabular}

The second test is done by the expert user, where the expert user is asked to determine the relevance of query with the hadith documents that has been retrieved by the system. The result of expert testing is given by Table 5 .

Table 5. Result of expert testing

\begin{tabular}{|l|c|c|c|c|c|}
\hline \multicolumn{7}{|c|}{ EXPERT TESTING RESULT } \\
\hline \multirow{2}{*}{ Query } & \multicolumn{2}{|c|}{ Ground Truth from Expert } \\
\cline { 2 - 7 } & $\mathbf{1}$ & $\mathbf{2}$ & $\mathbf{3}$ & $\mathbf{4}$ & $\mathbf{5}$ \\
\hline Q-1 & 28 & 29 & 47 & 22 & \\
\hline Q-2 & 9 & 6 & 25 & 24 & \\
\hline Q-3 & 29 & 30 & & & \\
\hline Q-4 & 38 & 47 & 48 & & \\
\hline Q-5 & 248 & 282 & & & \\
\hline Q-6 & 47 & 28 & 29 & 22 & 48 \\
\hline Q-7 & 69 & 70 & 55 & & \\
\hline Q-8 & 122 & 121 & 126 & 215 & 125 \\
\hline Q-9 & 8 & 369 & 113 & & \\
\hline Q-10 & 72 & 127 & 115 & 116 & 71 \\
\hline Q-11 & 134 & & & & \\
\hline Q-12 & 142 & 143 & 144 & 490 & \\
\hline Q-13 & 160 & & & & \\
\hline
\end{tabular}

\begin{tabular}{|l|c|c|c|c|c|}
\hline Q-14 & 163 & 164 & & & \\
\hline Q-15 & 169 & 168 & 170 & & \\
\hline Q-16 & 183 & 181 & 182 & & \\
\hline Q-17 & 195 & 194 & & & \\
\hline Q-18 & 207 & 206 & 208 & 209 & \\
\hline Q-19 & 381 & 380 & & & \\
\hline Q-20 & 161 & & & & \\
\hline Q-21 & 284 & & & & \\
\hline Q-22 & 53 & 364 & & & \\
\hline Q-23 & 260 & 264 & 265 & 266 & 263 \\
\hline Q-24 & 29 & 94 & 11 & 19 & \\
\hline Q-25 & 27 & & & & \\
\hline Q-26 & 149 & 148 & 150 & 154 & 153 \\
\hline Q-27 & 205 & & & & \\
\hline Q-28 & 50 & 51 & 24 & 25 & \\
\hline Q-29 & 217 & 218 & & & \\
\hline Q-30 & 135 & 136 & 176 & 159 & 141 \\
\hline Q-31 & 312 & 311 & 351 & & \\
\hline Q-32 & 220 & 275 & 234 & 217 & \\
\hline Q-33 & 400 & 398 & 402 & 401 & 403 \\
\hline Q-34 & 451 & 452 & 453 & & \\
\hline Q-35 & 480 & 420 & 447 & 414 & 477 \\
\hline Q-36 & 510 & 508 & 509 & 507 & 506 \\
\hline Q-37 & 432 & 433 & 428 & 431 & 439 \\
\hline Q-38 & 411 & 409 & 410 & & \\
\hline Q-39 & 338 & 339 & 341 & 340 & \\
\hline Q-40 & 303 & 302 & 304 & & \\
\hline Q-41 & 473 & 474 & 475 & 476 & 477 \\
\hline Q-42 & 155 & & & & \\
\hline Q-43 & 269 & 268 & 270 & 566 & 503 \\
\hline Q-44 & 517 & 515 & & & \\
\hline Q-45 & 162 & 166 & 189 & 154 & 163 \\
\hline Q-46 & 197 & 199 & 196 & 198 & \\
\hline Q-47 & 527 & 529 & 530 & 526 & 528 \\
\hline Q-48 & 406 & 407 & 408 & & \\
\hline Q-49 & 546 & 545 & 544 & & \\
\hline Q-50 & 58 & 85 & 534 & 59 & \\
\hline & & & & & \\
\hline
\end{tabular}

To evaluating the performance of applying the method to the system that has been made, the test is starting with comparing the result of system testing with the expert testing to measure the recall, precision, accuracy and f-measure. The test is carried out in 3 stages, the first stage is testing on top 5 data, then testing on top 3 data and testing on top 1 data. The test is carried out on the system without using query expansion and by using query expansion. The results of testing is given by Table 6 .

Table 6. Result of testing without query expansion

\begin{tabular}{|c|c|c|c|c|}
\hline \multicolumn{5}{|c|}{ Results of testing without query expansion } \\
\hline & Recall & Precision & Accuracy & F-Measure \\
\hline TOP 5 DATA & 79,81 & 51,2 & 68 & 59,12 \\
\hline TOP 3 DATA & 68,34 & 58,01 & 79,2 & 61,14 \\
\hline TOP 1 DATA & 62 & 62 & 92,4 & 62 \\
\hline
\end{tabular}

Based on Table 6 , in the top 5 data testing obtained an average recall value of $79.81 \%$, the average precision value of $51.2 \%$, the average accuracy value of $68 \%$ and the average f-measure value of $59.12 \%$, whereas in the top 3 data testing obtained an average recall value of $68.34 \%$, an average precision value of $58.01 \%$, an average accuracy value of $79.2 \%$ and an average fmeasure value of $61.14 \%$, and in the top 1 data testing obtained an average recall value of $62 \%$, the average precision value of $62 \%$, the average accuracy value of $92.4 \%$ and the average f-measure value of $62 \%$. The comparison graph based on the table above can be seen in Figure 2. 


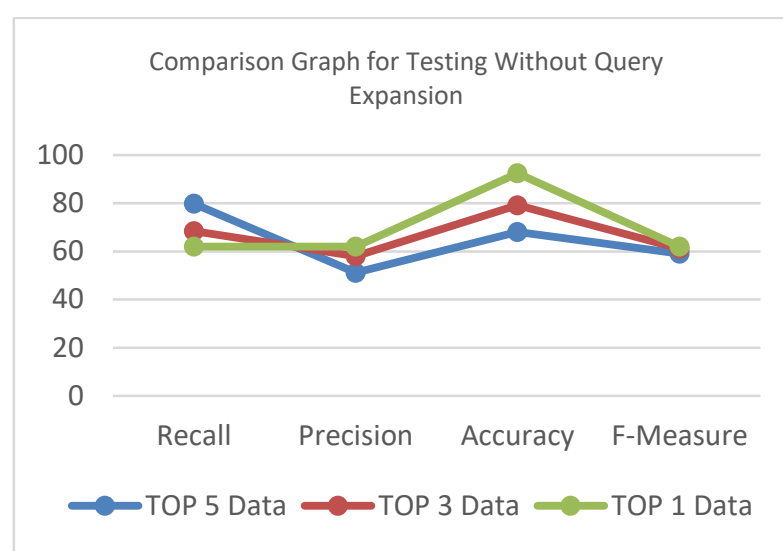

Figure 2. Comparison graph for testing without query expansion

And the result of testing with query expansion is given by Table 7 .

Table 7. Result of testing with query expansion

\begin{tabular}{|c|c|c|c|c|}
\hline \multicolumn{5}{|c|}{ Reasults of testing with query expansion } \\
\hline & Recall & Precision & Accuracy & F-Measure \\
\hline TOP 5 DATA & 81,97 & 53,2 & 70,08 & 61,18 \\
\hline TOP 3 DATA & 72,67 & 60,67 & 80,6 & 64,07 \\
\hline TOP 1 DATA & 66 & 66 & 93,2 & 66 \\
\hline
\end{tabular}

Based on Table 7 , in the top 5 data testing obtained an average recall value of $81.97 \%$, an average precision value of $53.2 \%$, an average accuracy value of $70.08 \%$ and an average f-measure value of $61.18 \%$, whereas in the top 3 data testing obtained an average recall value of $72.67 \%$, an average precision value of $60.67 \%$, an average accuracy value of $80.6 \%$ and an average fmeasure value of $64.07 \%$, and in the top 1 data testing obtained an average recall value of $66 \%$, an average precision value of $66 \%$, an average accuracy value of $93.2 \%$ and an average f-measure value of $66 \%$. The comparison graph based on the table above is given by Figure 3.

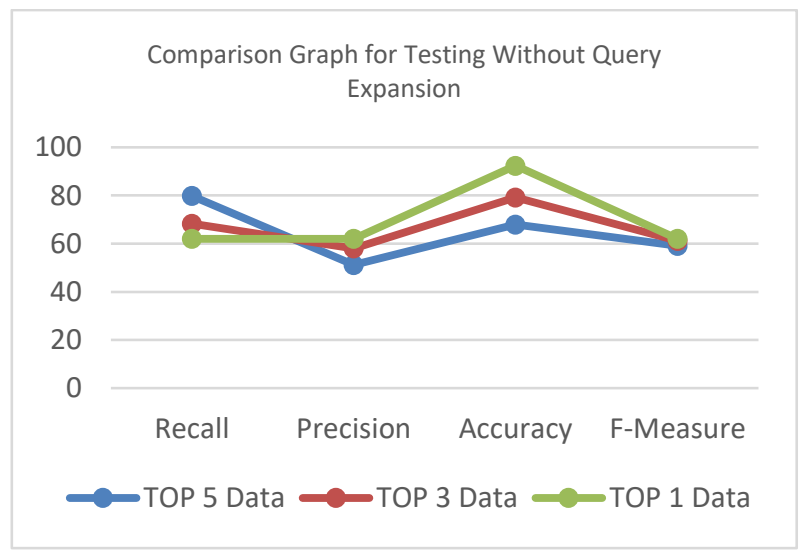

Figure 3. Comparison graph for testing with query expansion

\section{ACKNOWLEDGMENT}

Based on the results of the implementation and testing that have been conducted by the researcher, get the conclusion: based on testing of the top 5 data the use of query expansion shows an improvement in the average recall of $2.16 \%$, an improvement in the average precision of $2 \%$, an improvement in the average accuracy of $2.08 \%$, and an improvement in the average f-measure of $2.06 \%$ compared by without using query expansion. Moreover, based on testing of the top 3 data the use of query expansion shows an improvement in the average recall of $4.33 \%$, an improvement in the average precision of $2.66 \%$, an improvement in the average accuracy of $1.4 \%$ and an improvement in the average f-measure of $2.93 \%$ compared by without using query expansion. Finally, based on testing of the top 1 data the use of query expansion shows an improvement in the average recall of $4 \%$, an improvement in the average precision of $4 \%$, an improvement in the average accuracy of $0.8 \%$ and an improvement in the average $\mathrm{f}$ measure of $4 \%$ compared by without using query expansion.

Based on the explanation about the results of the implementation above, it can take a conclusion that the use of Indonesian thesaurus based on query expansion could give the better result than without using query expansion.

\section{REFERENCES}

[1] Baqi, M. F. (2015). AL Lu' Lu' Wal Marjan, Muttafaqun 'Alaih Shahih Bukhari. Solo: Beirut.

[2] Ginting, S. L., \& Trinada, R. P. (2014). Teknik Data Mining Menggunakan Metode Bayes Classifier Untuk Optimalisasi Pencarian Pada Aplikasi Perpustakaan. JATI UNIKOM, 14.

[3] Yadav, D. P. (2014). Document-Document Similarity Matrix and Naive-Bayes Classification to Web Information Retrieval. International Journal of Engineering Research and General Science Volume 2, 7.

[4] Shahabadkar, D. R., Reddy, Y. V., Khrisna, B. M., \& \& Devi, T. (2017). Enhanced Query Expansion For Web Information Retrieval. International Journal of Civil Engineering and Technology (IJCIET). International Journal of Civil Engineering and Technology (IJCIET), Volume 8, Issue 8, 6.

[5] Suyanto, S. M. (2017). Data Mining Untuk Klasifikasi Dan Klasterisasi Data. Bandung: INFORMATIKA.

[6] Hasugian. (2006). Penggunaan bahasa alamiah dan kosa kata terkendali dalam sistem temu balik informasi berbasis teks, departemen studi perpustakaan dan informasi universitas sumatera utara. Medan: Pustaka. 